\title{
Enhanced Tracheostomy Wound Healing Using Maltodextrin and Silver Alginate Compounds in Pediatrics: A Pilot Study
}

\author{
Larry D Hartzell MD, Tara N Havens, Brian H Odom MSPT CWS, Tanya G Stillman RN, \\ Jessica L Boswell CRS, Charles M Bower MD, and Gresham T Richter MD
}

\begin{abstract}
BACKGROUND: Tracheostomy wounds are commonly encountered in children but rarely reported. Relatively few treatments are available or have been investigated to manage this problem. Healing times for pediatric tracheostomy wounds are often unpredictable and protracted. Recent use of maltodextrin gel (MD) and a silver alginate sponge (AG) at our institution has demonstrated expedited healing and interest in this novel treatment for tracheostomy wounds. METHODS: We conducted an 11-month retrospective review of children with wound complications following tracheostomy placement at a tertiary care facility. Wounds were evaluated and rated based upon the National Pressure Ulcer Advisory Panel staging system. Subjects identified with stage 2 or greater tracheostomy-related ulcers treated with $\mathrm{MD}$ and/or AG were included. Subject characteristics and wound healing rates were tabulated in a database that included age, wound site, initial and final wound stage, type of treatment, length of treatment, and complications. Tracheostomy wounds treated as an out-patient were excluded from the study. RESULTS: Eighteen subjects, which included both in-patients and out-patients, were treated with AG and/or MD during the study period for tracheostomy-related wounds. Of the 26 subjects with tracheostomies performed during the study period, $10(38.5 \%)$ were treated for postoperative wounds. A total of 11 subjects completed their in-patient wound treatment and were thus included in the study. Average subject age was $5.3 \mathrm{y}$ (range $0.25-15.6 \mathrm{y}$ ). Wound locations were as follows: infrastomal region $(n=8)$, stoma $(n=2)$, and lateral neck $(n=1)$. Six subjects had stage 2 wounds, 4 had stage 3 wounds, and 1 had a stage 4 ulcer. All wounds achieved complete epithelialization following treatment with MD and/or AG. The average length of treatment was $12.8 \mathrm{~d}$ (range 6-28 d). No adverse effects were identified. CONCLUSIONS: Postoperative tracheostomy wounds are common. The use of MD and AG provides an effective and safe treatment for tracheostomy-related ulcers. Key words: tracheostomy; wound care; pressure ulcer; Multidex; maltodextrin; Algidex; silver alginate; pediatric; complication; wound; ulcer. [Respir Care 2014;59(12):1857-1862. (C) 2014 Daedalus Enterprises]
\end{abstract}

\section{Introduction}

Tracheostomy is a common procedure performed for the management of upper and lower airway conditions. This technique is performed on both children and adults with some minor differences in technique and indications. ${ }^{1,2}$

Drs Hartzell, Bower and Richter are affiliated with the Department of Otolaryngology-Head and Neck Surgery, Division of Pediatric Otolaryngology, University of Arkansas for Medical Sciences, Arkansas Children's Hospital; Ms Havens is affiliated with the College of Medicine, University of Arkansas for Medical Sciences; Mr Odom is affiliated with the Physical Therapy Department, Division of Wound Care, Arkansas Children's Hospital; Ms Stillman is affiliated with the Otolaryngology Department, Arkansas Children's Hospital; Ms Boswell is affiliated with
Many studies have looked at outcomes of tracheostomies, reporting on the mortality rates and major complications such as hemorrhage, dislodgement, and pneumothorax. ${ }^{3-5}$

\footnotetext{
the Department of Otolaryngology-Head and Neck Surgery, Division of Pediatric Otolaryngology, Arkansas Children's Hospital, Little Rock, Arkansas.

The authors have disclosed no conflicts of interest.

Drs Hartzell, Odom, Stillman, Bower and Richter presented a version of this paper at the at American Society of Pediatric Otolaryngology Annual Meeting, held April 29 to May 1, 2011, in Chicago, Illinois.
}

DOI: $10.4187 /$ respcare. 02822 
Only a few studies have included wound healing in their series of tracheostomy-related complications. ${ }^{6}$ Regardless, wounds related to pediatric tracheostomies are common. Jaryszak and colleagues ${ }^{7}$ found a pediatric tracheostomy wound complication rate of $29 \%$. They further demonstrated a higher incidence (39\%) in children younger than 12 months of age. ${ }^{7}$ This rate is higher than that previously reported by Kremer and colleagues, ${ }^{6}$ who estimated a rate of $1-15 \%$ in a meta-analysis. Nonetheless, the highest rates of complications were also found by this group to be in premature infants and neonates.

Tracheostomy wounds and pressure ulcers most frequently occur in the immediate postoperative period but may also be discovered during out-patient follow-up. Tracheostomyrelated wounds may occur despite healthcare vigilance, preventive management, and early intervention. These wounds have a relatively high incidence in children compared with adults, as summarized by Corbett et al, ${ }^{4}$ with the morbidity and mortality associated with tracheostomy being 2-3 times higher in children than adults. Prevention of hospital-acquired and device-related wounds is an area of increasing interest. A study by Boesch and colleagues ${ }^{8}$ addressed the prevalence of pressure ulcers directly related to tracheostomies. They found a significant reduction in wounds after implementing a few specific practices such as customized tubes, barrier devices, and close vigilance.

Although prevention of tracheostomy wounds is ideal for patient comfort and care, some wounds and other complications will inevitably occur. No standardized treatment for tracheostomy-related wounds, however, has been established in the literature. Most care providers have traditionally managed these wounds with the use of barrier gauze or sponges and at times with antibiotic ointment or creams. ${ }^{7}$ Healing, however, can take an extended period of time, with significant cost and inconvenience to the patient and caregiver.

The use of maltodextrin gel (MD); (Multidex, DeRoyal Industries, Powell, Tennessee) and silver alginate foam pads (AG); (Algidex Ag, DeRoyal Industries) is a novel approach to managing perioperative wounds and pressure ulcerations. The use of these agents was introduced for the care of tracheostomy-related wounds at our institution in December 2009. Soon thereafter, the wound care team and tracheostomy specialty nurses recognized great improvement in the speed and degree of healing of tracheostomy ulceration. With this important albeit anecdotal evidence, this study was created to explore the early experience and safety with MD and AG foam pads for tracheostomy wound care during the first year of its systematic application.

\section{Methods}

The institutional review board at the University of Arkansas for Medical Sciences approved this study. A retrospective chart review was performed. The charts of all

\section{QUICK LOOK}

\section{Current knowledge}

Tracheostomy wounds are commonly encountered in children. Few treatments are available or investigated to manage this difficult problem. Healing times for pediatric tracheostomy wounds are unpredictable and protracted.

\section{What this paper contributes to our knowledge}

Postoperative tracheostomy wounds are common complications. The use of maltodextrin gel (MD) and silver alginate foam pads (AG) provided an effective treatment for tracheostomy-related ulcers in pediatric patients.

subjects who underwent tracheostomy by the otolaryngology service at a tertiary care facility (Arkansas Children's Hospital) from December 29, 2004 until November 2, 2010 were reviewed. Additionally, the charts of all subjects who were treated between December 29, 2009 and November 2, 2010 with AG and/or MD for tracheostomyrelated wound complications were reviewed. Subjects who were treated with $\mathrm{AG}$ and/or MD as out-patients were then excluded from the study to prevent technical bias and provide clear data with documented and consistent wound care in the study population.

Records reviewed and tabulated included the initial consultation, operative reports, and daily in-patient progress notes. A database including subject demographics, history, age, wound location, wound stage, treatment regimen, length of treatment, outcome, and complications was created. Stage of wound was determined based on the 2007 National Pressure Ulcer Advisory Panel guidelines. ${ }^{9}$ Records of tracheostomy patients for the $5 \mathrm{y}$ before the study period (December 29, 2009 through November 2, 2010) were reviewed in an effort to establish a control group for the study population.

\section{Results}

A total of 18 subjects were treated with AG and/or MD for tracheostomy wounds during the study period from December 29, 2009 through November 2, 2010. This included 2 subjects who were transferred to the study facility with prior tracheostomies, as well as 6 subjects who received all of their treatment as out-patients. Ten out of 26 subjects (38.5\%) who underwent tracheostomy at the study facility during this period were identified as having postoperative tracheostomy-related wounds. After combining these groups, a total of 11 subjects both started and com- 


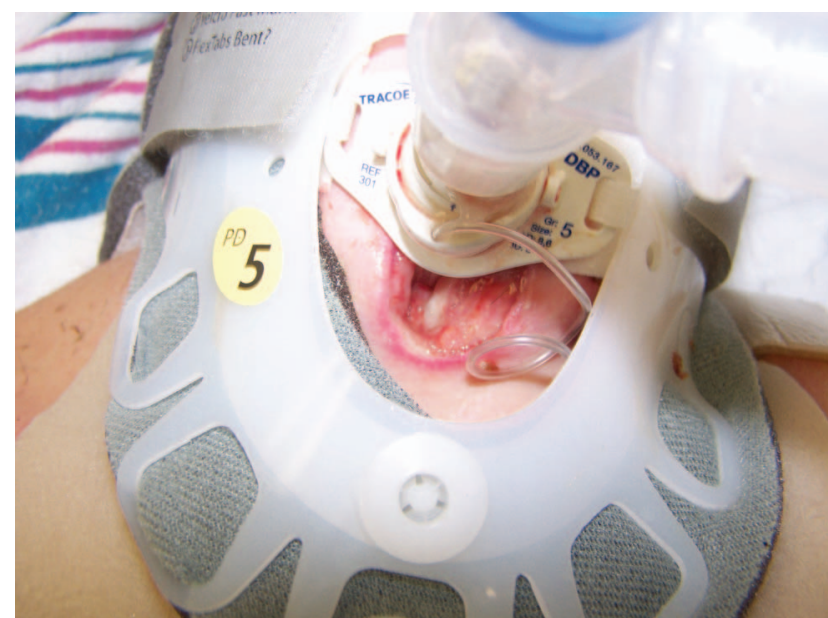

Fig. 1. Subject 6. Stomal and infrastomal pressure ulcer and operative wound before treatment.

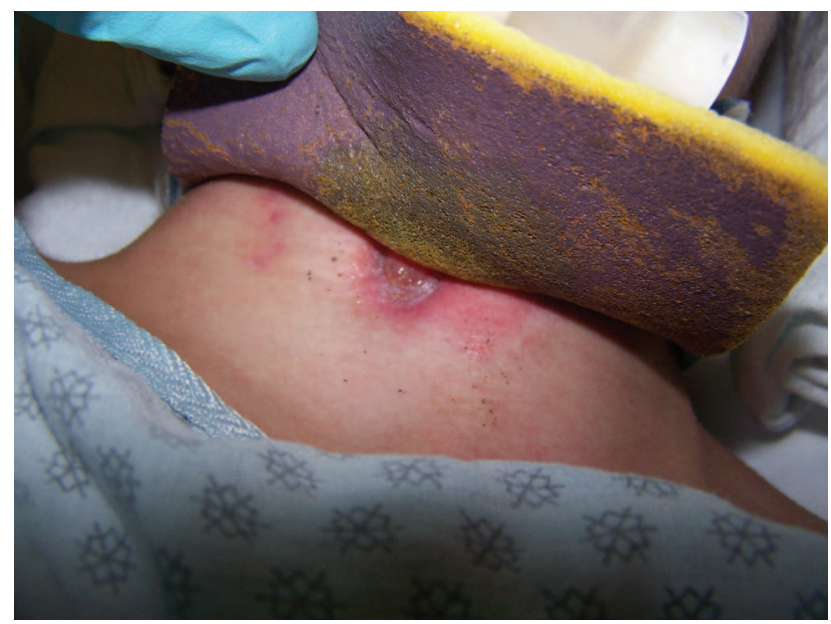

Fig. 2. Subject 6 after $28 \mathrm{~d}$ of silver alginate sponge + maltodextrin gel.

pleted their treatment as in-patients and were thus included in the study population. The average subject age was $5.3 \mathrm{y}$, with a range from 0.25 to $15.6 \mathrm{y}$ (median $3.2 \mathrm{y}$; interquartile range $0.75-12.2 \mathrm{y}$ ). The gender distribution for this same group was skewed toward male predominance with 9 male and 2 female subjects.

There were 3 types of wounds in the group, as shown in Figures 1-6. Of the 11 subjects included in the study group, 8 had infrastomal wounds (Fig. 3), 2 had stoma wounds (Fig. 1), and 1 had a lateral neck ulcer (Fig. 5). This is detailed in Table 1. Initial wound stage for this group is summarized in Table 1 . This included stage 2 $(n=6)$, stage $3(n=4)$, and stage $4(n=1)$ ulcers based on the 2007 National Pressure Ulcer Advisory Panel guidelines (see Table 2). ${ }^{9}$

The average length of treatment for the study group was $12.8 \mathrm{~d}$, with a range from 6 to $28 \mathrm{~d}$ (median $\pm \mathrm{SD}$, $10.0 \pm 7.6 \mathrm{~d}$ ). Nine of 11 subjects had the combination of

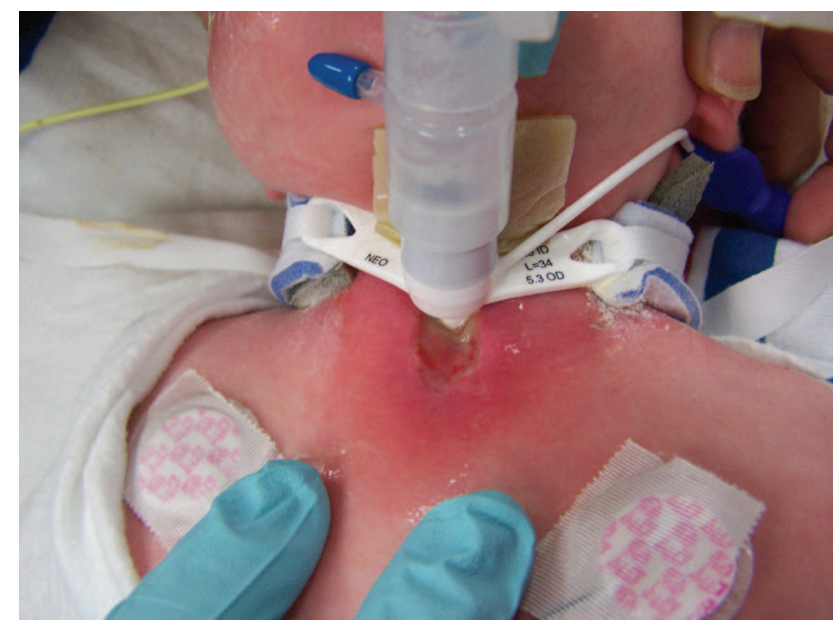

Fig. 3. Subject 5. Infrastomal pressure ulcer before treatment.

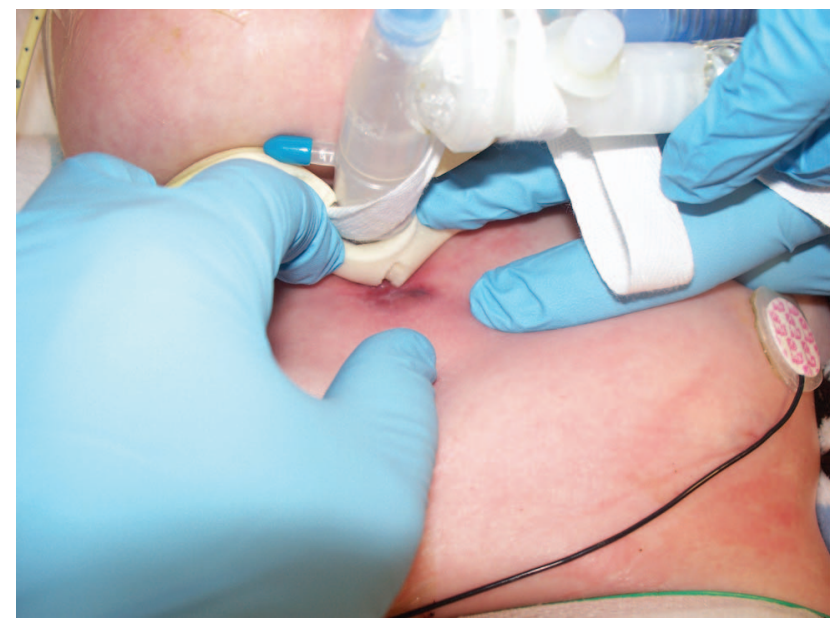

Fig. 4. Subject 5 after $10 \mathrm{~d}$ of silver alginate sponge + maltodextrin gel.

MD and AG treatments, and 2 subjects were treated with MD alone (see Table 1). Each of the 11 subjects' wounds achieved complete epithelialization by completion of treatment (see Figs. 2, 4, and 6).

No adverse effects from the MD and AG treatments were identified.

A total of 188 subjects were identified as having received tracheostomies by the otolaryngology service at the study institution during the study period (December 29, 2009 through November 2, 2010) and the 5 y preceding (December 29, 2004 through December 28, 2009). Unfortunately, during the $5 \mathrm{y}$ before AG and MD were utilized for wound care, tracheostomy site surveillance, wound documentation, and wound care were not routinely recorded. Comparative data were thus unavailable to provide a reliable control group for the study population. For example, only 5 subjects outside the study period were documented as having tracheostomy wounds that required treatment. 


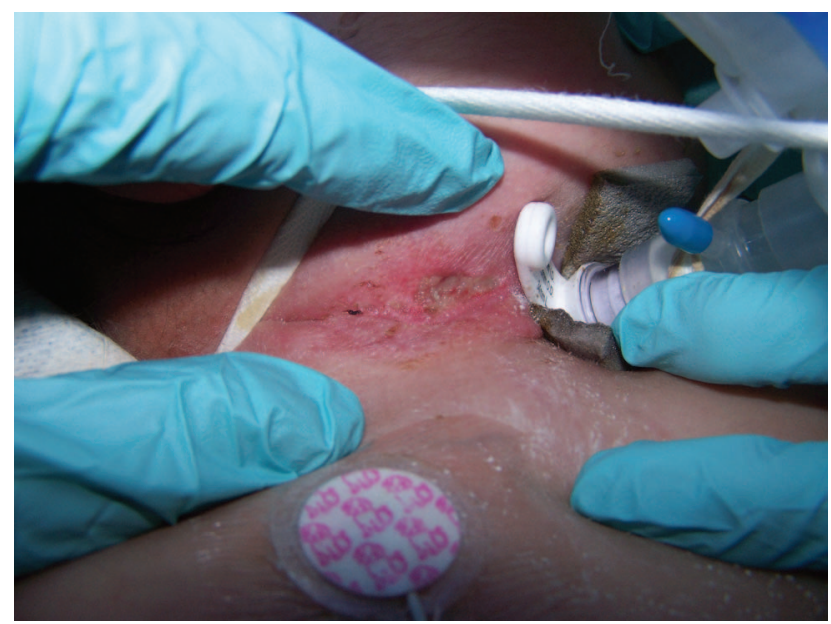

Fig. 5. Subject 4. Right lateral neck pressure ulcer before treatment.

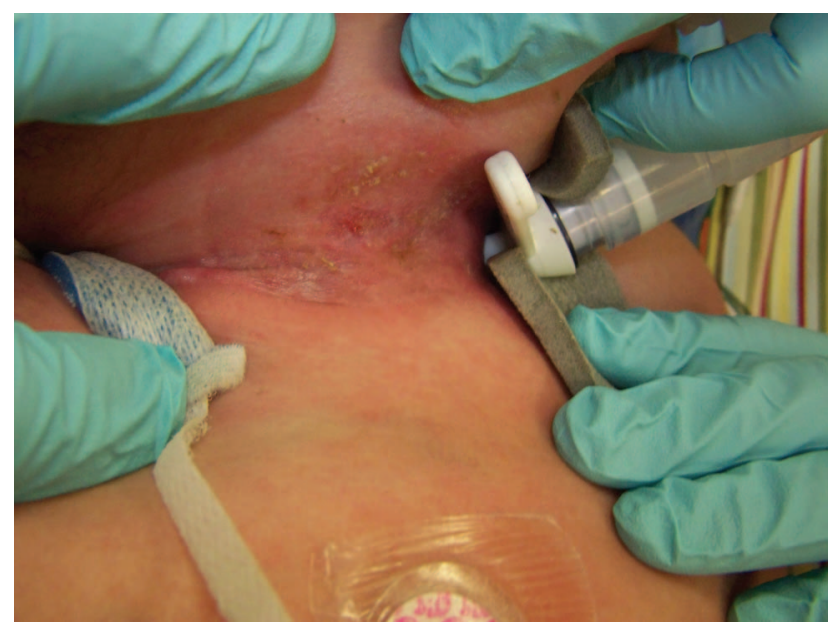

Fig. 6. Subject 4 after $3 \mathrm{~d}$ of silver alginate sponge + maltodextrin gel.

Of these, 3 subjects had wounds documented for several months with no clear resolution. Another subject's wound lasted for 1 month, and the other subject's lasted for 2 weeks using a variety of methods for wound care. Therefore, this information was not included in the data analysis, and statistical comparisons of treatment outcomes could not be formulated.

\section{Discussion}

In the pediatric population, pressure- and frictionrelated wounds secondary to the tracheostomy tube and ties are frequently encountered and require special attention. Prevention and early detection are the cornerstones to effective tracheostomy wound care, as described by Boesch et al. ${ }^{8}$ We currently follow similar guidelines at our facility in the management of all patients with tracheostomy tubes. However, despite these preventive efforts, wounds
Table 1. Subjects Treated With Maltodextrin and Silver Alginate Compounds

\begin{tabular}{rrcccc}
\hline \hline Patient & $\begin{array}{c}\text { Age } \\
(\mathrm{y})\end{array}$ & $\begin{array}{c}\text { Wound } \\
\text { Location }\end{array}$ & $\begin{array}{c}\text { Wound } \\
\text { Stage }\end{array}$ & Treatment & $\begin{array}{c}\text { Duration } \\
(\mathrm{d})\end{array}$ \\
\hline 1 & 15.4 & Infrastoma & 2 & $\mathrm{MD}+\mathrm{AG}$ & 8 \\
2 & 3.8 & Infrastoma & 2 & $\mathrm{MD}+\mathrm{AG}$ & 14 \\
3 & 3.2 & Infrastoma & 2 & $\mathrm{MD}+\mathrm{AG}$ & 6 \\
4 & 0.9 & Lateral neck & 3 & $\mathrm{MD}+\mathrm{AG}$ & 21 \\
5 & 0.3 & Infrastoma & 3 & $\mathrm{MD}+\mathrm{AG}$ & 10 \\
6 & 12.2 & Stoma and infrastoma & 4 & $\mathrm{MD}+\mathrm{AG}$ & 28 \\
7 & 15.6 & Stoma and infrastoma & 3 & $\mathrm{MD}+\mathrm{AG}$ & 22 \\
8 & 0.8 & Infrastoma & 3 & $\mathrm{MD}+\mathrm{AG}$ & 12 \\
9 & 0.5 & Infrastoma & 2 & $\mathrm{MD}$ & 6 \\
10 & 5.7 & Infrastoma & 2 & $\mathrm{MD}$ & 6 \\
11 & 0.3 & Infrastoma & 2 & $\mathrm{MD}+\mathrm{AG}$ & 8
\end{tabular}

$\mathrm{MD}=$ maltodextrin

$\mathrm{AG}=$ silver alginate foam

Table 2. 2007 National Pressure Ulcer Advisory Panel Staging System

\begin{tabular}{|c|c|}
\hline Stage & Description \\
\hline I & Intact skin with non-blanchable redness of a localized area. \\
\hline II & $\begin{array}{l}\text { Partial thickness loss of dermis, presenting as a shallow } \\
\text { open ulcer with a red-pink wound bed without slough. }\end{array}$ \\
\hline III & $\begin{array}{l}\text { Full thickness tissue loss. Subcutaneous fat may be visible } \\
\text { but not bone (cartilage), tendon, or muscle. }\end{array}$ \\
\hline IV & $\begin{array}{l}\text { Full thickness tissue loss with exposed bone (cartilage), } \\
\text { tendon, or muscle. }\end{array}$ \\
\hline
\end{tabular}

related to tracheostomy tubes still occur in pediatric patients. Hypothetically, this may be due to their comparatively shorter necks, increased wet environment from secretions and perspiration, frequent and rapid head and neck movements compared with more sedentary adults, and the routine use of firm and inflexible methods to secure the tube to prevent accidental decannulation. All of these factors likely lead to areas of pressure and friction that may result in open wounds.

Despite this common occurrence, tracheostomy-related wounds have no standardized treatment, and very little can be found in the literature about management of these unique wounds. Of the few articles that address the topic of tracheostomy-related wounds, the occurrence of wounds is mentioned, but specific treatments administered, duration of treatment, or recommendations are not described. ${ }^{3-7} \mathrm{Al}-$ though sponge and gauze barriers present viable solutions, the healing course is often lengthy and unpredictable and sometimes complete healing is not achieved. As these wounds lie in close proximity to the respiratory tract, it is 


\section{Tracheostomy Wound Healing}

important to also bear in mind the absorptive properties inherent in this region and to pay careful attention to the treatment options considered.

Silver-containing compounds have been utilized extensively for wound care in both adults and children. Multiple studies have described the bactericidal properties of silver. ${ }^{10-12}$ In addition to the antibacterial properties of silver, there are some additional studies that have analyzed the anti-inflammatory actions of silver and the benefits this may have with wound healing. ${ }^{13,14}$ Algidex Ag is a foam pad coated with silver alginate in a maltodextrin matrix, and performed very well in its bactericidal effects and the duration of treatment when compared with other silvercontaining dressings. ${ }^{15-18}$ The embedded silver is in an ionic state, providing for more rapid and continued release to the underlying tissue. The release of silver ions has been found to be at a fairly constant rate over a total of $10 \mathrm{~d}$, although the pads are recommended to be changed after 7 d. ${ }^{17}$

The safety of silver usage in both infants and adults has also been thoroughly investigated. ${ }^{12,17-19}$ Khattak et al ${ }^{17}$ looked specifically at the use of AG in very low birthweight infants and found that serum concentrations of silver were well below toxic levels, and there was no evidence of serum accumulation of silver or effects on the hepatic or renal systems.

Although silver has been utilized ubiquitously in wound care, maltodextrin and related sugars have not been as widely employed. However, the benefit of maltodextrin for wound healing and its mechanisms have been described. ${ }^{20-23} \mathrm{MD}$ is a mixture of monosaccharides and polysaccharides. Effectively, MD serves as a chemotactant for white blood cells, macrophages, and fibroblasts. ${ }^{22}$ This provides wound debridement and antibacterial effects. The influx of fibroblasts also promotes collagen formation, which is critical for healing. The gel form also provides the wound with a moist environment, which has been shown to be beneficial for healing. ${ }^{24}$

Both products, AG and MD, effectively eliminate infection and promote granulation tissue to assist in the healing process. The AG product also has the added benefit of serving as a barrier pad, which relieves pressure from the tube and its associated ties. The location of tracheostomy wounds has always presented an interesting dilemma to wound care. Due to the increased absorptive capabilities of the trachea, the usage of materials that can cause toxicity or potential irritation are avoided. Both MD and AG have been found to be safe for use in this delicate region. We identified no adverse effects from the use of these products for the subjects included in this retrospective study.

Although both AG and MD have been described as safe and effective in wound care, no reports of the use of AG or MD for tracheostomy wounds are found in the literature. After approval through the products evaluation com- mittee at Arkansas Children's Hospital, we started using these products in late 2009 and quickly noticed an anecdotal remarkable decrease in length of treatment for tracheostomy-related wounds with an average time to heal of $12.8 \mathrm{~d}$ (median duration was $10 \mathrm{~d}$ ) in the 11 cases included in this study, as compared with the typical weeks-tomonths' time frame we experienced with other methods (such as gauze or foam barriers, ointments including scarlet red, and even lip balm). Because of the notable improvement in wound management with $\mathrm{AG}$ and $\mathrm{MD}$, nearly all patients from that time forward have been treated with a combination of these products.

Improved reporting and documentation of wound progress also started during this time, compared with the sporadic and inconsistent records maintained previously. In an effort to obtain comparative data with these other treatment options, our group looked at all patients who had a tracheostomy performed at our institution by the otolaryngology service during the $5 \mathrm{y}$ before initiation of MD and AG use, as well as those performed during the study period.

We found a total of 188 tracheostomies performed by the otolaryngology service at the study facility during a 6-y period. Twenty-six were during the period when AG and MD were being used. Wounds were discovered in 10 of these subjects, in addition to another 2 subjects who received their tracheostomy at an outside facility and were found to have wounds after transfer. One of the 12 subjects treated with $\mathrm{AG}$ and/or MD as in-patients was discharged before completing treatment and was not included in the study data.

Out of the 162 patients with tracheostomies performed during the $5 \mathrm{y}$ before the use of $\mathrm{AG}$ and $\mathrm{MD}$, only 5 were documented within the in-patient records as having tracheostomy-related wounds that required management or wound care. Unfortunately, this is likely a vast underestimation of postoperative tracheostomy wound incidence. This deficiency can likely be attributed to such factors as: incomplete documentation, lack of appreciation for the prevalence and significance of postoperative tracheostomy wounds, and inaccessibility to a wound care service during much of the period. There were many wounds that occurred during this time that were treated according to the memory of the various care providers, but these were unfortunately not documented or reported. Therefore, a comparison group to our study group was unable to be identified and used.

Despite using routine, common methods, tracheostomy wounds occurred. An incidence of 10 postoperative ulcerations were identified out of 26 tracheostomies performed during the study period (38.5\%), which falls in line with the rates reported in previous studies. ${ }^{4,6,7}$

Fortunately, due to a heightened awareness, the establishment of tracheostomy and wound care protocols, and 
the addition of specially trained individuals both within our department and our institution, these wounds are now being recognized and reported much earlier. Tracheostomy wounds are also now monitored very closely, and greater efforts at prevention are taking place. ${ }^{8}$

In this study, 9 out of 11 subjects were treated with the combined use of MD and AG. The 2 subjects treated with MD alone had stage 2 ulcers that were small and healed quickly. This raises the question of whether the combined use of MD and AG is necessary for each wound or whether their use in isolation may be just as effective. Furthermore, the question remains whether similar products can achieve comparable results. Future studies may help elucidate this question.

This pilot study demonstrates that the use of AG and MD for tracheostomy-related wounds is effective for tracheostomy wound healing. This has developed into the new standard of care at our institution. All wounds managed with these treatments in this study completely healed and did not require removal of the tracheostomy tube or surgical intervention.

Tracheostomy wound reporting and care are scarcely encountered in the published literature. Although complications from tracheostomies have been described, little has been discussed as to how to care for these unique wounds. This is clearly an area that would benefit from additional attention.

We recognize the limitations of our study, which are inherent to a retrospective study. The lack of a control group makes it difficult to establish firm recommendations and statements regarding proven efficacy and added benefit over other available treatments. Generalized recommendations cannot be made from this study due to lack of a comparison group. However, we present this technique as an effective and viable option for tracheostomy wound care. We hope to clarify some of the questions regarding the objective benefits of this treatment regimen with prospective trials that are being pursued. We also feel that this study helps increase the awareness of wound problems that are commonly associated with tracheostomies and offers some options for effective management.

\section{Conclusions}

Tracheostomy wounds are common, especially within the pediatric population. The novel use of MD and AG is both a safe and effective treatment option for pediatric tracheostomy-related wounds.

\section{REFERENCES}

1. Wetmore RF, Handler SD, Potsic WP. Pediatric tracheostomy: experience during the past decade. Ann Otol Rhinol Laryngol 1982; 91(6 Pt 1):628-632.

2. Gilmore BB, Jr., Mickelson SA. Pediatric tracheotomy. Controversies in management. Otolaryngol Clin North Am 1986;19(1):141-151.
3. Carr MM, Poje CP, Kingston L, Kielma D, Heard C. Complications in pediatric tracheostomies. Laryngoscope 2001;111(11 Pt 1):19251928.

4. Corbett HJ, Mann KS, Mitra I, Jesudason EC, Losty PD, Clarke RW. Tracheostomy: a 10-year experience from a UK pediatric surgical center. J Pediatr Surg 2007;42(7):1251-1254.

5. Al-Samri M, Mitchell I, Drummond DS, Bjornson C. Tracheostomy in children: a population-based experience over 17 years. Pediatr Pulmonol 2010;45(5):487-493.

6. Kremer B, Botos-Kremer AI, Eckel HE, Schlöndorff G. Indications, complications, and surgical techniques for pediatric tracheostomies: an update. J Pediatr Surg 2002;37(11):1556-1562.

7. Jaryszak EM, Shah RK, Amling J, Peña MT. Pediatric tracheotomy wound complications: incidence and significance. Arch Otolaryngol Head Neck Surg 2011;137(4):363-366.

8. Boesch RP, Myers C, Garrett T, Nie A, Thomas N, Chima A, et al. Prevention of tracheostomy-related pressure ulcers in children. Pediatrics 2012;129(3):e792-e797.

9. Black J, Baharestani MM, Cuddigan J, Dorner B, Edsberg L, Langemo D, et al. National Pressure Ulcer Advisory Panel's updated pressure ulcer staging system. Adv Skin Wound Care 2007;20(5): 269-274.

10. Driver VR. Silver dressings in clinical practice. Ostomy Wound Manage 2004;50(9A Suppl):11S-15S.

11. Ovington LG. The truth about silver. Ostomy Wound Manage 2004; 50(9A Suppl):1S-10S.

12. Madeo M, Martin CR, Turner C, Kirkby V, Thompson DR. A randomized trial comparing Arglaes (a transparent dressing containing silver ions) to Tegaderm (a transparent polyurethane dressing) for dressing peripheral arterial catheters and central vascular catheters. Intensive Crit Care Nurs 1998;14(4):187-191.

13. Lansdown AB. Silver. 1: its antibacterial properties and mechanism of action. J Wound Care 2002;11(4):125-130.

14. Lansdown AB. Silver. 2: toxicity in mammals and how its products aid wound repair. J Wound Care 2002;11(5):173-177.

15. Thomas S, McCubbin P. An in vitro analysis of the antimicrobial properties of 10 silver-containing dressings. J Wound Care 2003; 12(8):305-308.

16. Thomas S, McCubbin P. A comparison of the antimicrobial effects of four silver-containing dressings on three organisms. J Wound Care 2003;12(3):101-107.

17. Khattak AZ, Ross R, Ngo T, Shoemaker CT. A randomized controlled evaluation of absorption of silver with the use of silver alginate (Algidex) patches in very low birth weight (VLBW) infants with central lines. J Perinatol 2010;30(5):337-342.

18. Meaume S, Vallet D, Morere MN, Téot L. Evaluation of a silverreleasing hydroalginate dressing in chronic wounds with signs of local infection. J Wound Care 2005;14(9):411-419.

19. Hill ML, Baldwin L, Slaughter JC, Walsh WF, Weitkamp JH. A silver-alginate-coated dressing to reduce peripherally inserted central catheter (PICC) infections in NICU patients: a pilot randomized controlled trial. J Perinatol 2010;30(7):469-473.

20. Hogge J, Krasner D, Nguyen H, Harkless LB, Armstrong DG. The potential benefits of advanced therapeutic modalities in the treatment of diabetic foot wounds. J Am Podiatr Med Assoc 2000;90(2):57-65.

21. McFadden EA. Multidex gel for use in wound care. J Pediatr Nurs 1997;12(2):125.

22. Silvetti AN. An effective method of treating long-enduring wounds and ulcers by topical applications of solutions of nutrients. J Dermatol Surg Oncol 1981;7(6):501-508.

23. Stashak TS, Farstvedt E, Othic A. Update on wound dressings: indications and best use. Clin Tech Equine Pract 2004;3(2):148-163.

24. Junker JP, Caterson EJ, Eriksson E. The microenvironment of wound healing. J Craniofac Surg 2013;24(1):12-16. 